\title{
Optical and phonon excitations of modified Pandey chains at the $\operatorname{Si}(111)-2 \times 1$ surface
}

\author{
Charles H. Patterson, Soumya Banerjee, and John F. McGilp \\ School of Physics, Trinity College Dublin, Dublin 2, Ireland \\ (Received 22 July 2011; revised manuscript received 29 September 2011; published 18 October 2011)
}

\begin{abstract}
The modified Pandey $\pi$-bonded chain structure for the $(2 \times 1)$ reconstruction of the $\mathrm{Si}(111)$ surface has two nearly degenerate structures in which one or the other of two atoms in the $\pi$ chain is the outermost atom at the surface. Recent experimental data suggest that both structures can be found on a single $\mathrm{Si}(111)$ surface. Surface structures, reflectance anisotropy spectra, and the phonon contribution to the surface conductivity are calculated using a hybrid density functional theory (DFT) method. The modified Pandey chain with a positive tilt of the atoms in the $\pi$ chain has the lowest energy. A phonon around $50 \mathrm{meV}$ polarized parallel to the $\pi$ chains has anomalously large, anisotropic Born effective charges and dominates the conductivity. The hybrid DFT approach is suitable for calculating excitations in complex surface and interface structures with large unit cells.
\end{abstract}

DOI: 10.1103/PhysRevB.84.155314 PACS number(s): 78.40.Fy, 78.30.Am, 73.20.At, 78.68.+m

\section{INTRODUCTION}

The Pandey $\pi$-bonded chain model $^{1}$ for the $(2 \times 1)$ reconstruction of the $\mathrm{Si}(111)$ surface is one of the best known semiconductor surface reconstructions and is a good model system for testing new approaches for calculating the electronic, optical, and vibrational properties of surfaces. Its atomic structure has been computed from low-energy electron diffraction (LEED) data ${ }^{2-4}$ and using density functional theory (DFT) total energy methods. ${ }^{5-8}$ In the original model, ${ }^{1}$ atoms in the $\pi$ chain lie in the same horizontal plane; however, this structure was shown to be incompatible with LEED data ${ }^{2}$ and a buckled $\pi$ chain model was proposed. The two silicon atoms in each unit cell of the $\pi$ chains are inequivalent, even in the Pandey model. Consequently, there are two inequivalent buckling geometries in which one or the other of the $\pi$-chain atoms is outermost. In either case, one filled and one vacant surface state is found. The filled state is localized on the outermost atom of the $\pi$ chain throughout most of the surface Brillouin zone and the vacant state is localized on the inner atom in the $\pi$ chain. The half-filled nature of the surface states results in symmetry breaking and gap formation and may be the driving force for buckling of the $\pi$ chains.

Early calculations on buckled, $\pi$-bonded chain models ${ }^{5,6}$ showed that the energy difference between either buckling geometry was small. A subsequent reanalysis ${ }^{4}$ of LEED data from Himpsel et al. ${ }^{2}$ referred to either buckling of the $\pi$ chains as having positive or negative tilts and called them positiveor negative-tilt modified Pandey chains ( \pm MP chains). We use the same nomenclature here; the structures and surface state band orbitals are shown in Fig. 1. The occurrence of nearly degenerate filled and vacant electronic states on adjacent atoms in the $\pi$ chains results in large charge transfers between atoms on the $\pi$ chains during ion motion or optical transitions and anomalously large scattering or absorption cross sections from specific phonons or optical transitions. The large charge transfer is also expected to lead to strong electron-phonon coupling; the importance of electron-electron and electron-phonon effects in this surface has been discussed previously. ${ }^{7,9-11}$

The high-resolution electron energy loss spectroscopy (HREELS) spectrum of the Si(111) surface was first reported by Ibach $^{12}$ in 1971. Subsequent experimental ${ }^{13}$ and theoretical analyses of the phonon spectrum of $\pi$-bonded chain surfaces $^{6,14-16}$ found that a single phonon strongly localized on the $\pi$-chain atoms dominated the surface conductivity in the IR region. The optical response of the surface was first probed using surface differential reflectance (SDR), ${ }^{17}$ and subsequently by reflectance anisotropy spectroscopy (RAS), ${ }^{18}$ both techniques showing that the $\pi$ chains have a very strong anisotropic response in the infra-red. An early calculation predicted such a response in this region. ${ }^{19}$ The electronic band structure has been probed using angle-resolved photoelectron spectroscopy (ARPES) ${ }^{20}$ and inverse photoemission spectrocopy (IPES). ${ }^{21,22}$ Quite recently a combined ARPES, scanning tunneling microscopy and spectroscopy (STM and STS), and $G W$ calculation study ${ }^{23}$ concluded that it was possible to observe the coexistence of \pm MP chains on $n$-type $\mathrm{Si}(111)-2 \times 1$ surfaces.

Most first-principles calculations of optical response properties of semiconductor surfaces are performed using DFT in the local density approximation (LDA) or generalized gradient approximation (GGA) using plane-wave basis sets. It is well known that DFT underestimates band gaps of semiconductors and consequently predicts optical spectra of semiconductors incorrectly; this is corrected either by performing a perturbative $G W$ calculation before the optical response calculation or simply by shifting conduction bands upward by a constant amount. In some cases the Bethe-Salpeter equation (BSE) is solved in order to include electron-hole attraction in the excited state Hamiltonian and this may reduce the energy required for an optical excitation. ${ }^{10}$ In a perturbative $G W$ calculation the LDA or GGA exchange-correlation potential from the DFT self-consistent field calculation is replaced by a Fock exchange term screened by the dielectric function. In a narrow-gap semiconductor such as $\mathrm{Si}$, the extent of screening of Fock exchange in a $G W$ calculation is large at small wave vectors, so the wave vector and energy-dependent weight of Fock exchange is small. The predicted band gap of a material depends strongly on Fock exchange; (unscreened) Hartree-Fock theory overestimates the band gap of Si by more than $5 \mathrm{eV}^{24}$

Here we use a hybrid DFT method, with a Gaussian local orbital basis and the CRYSTAL ${ }^{25}$ and EXCITON ${ }^{24}$ codes. The exchange-correlation potential used throughout (see 


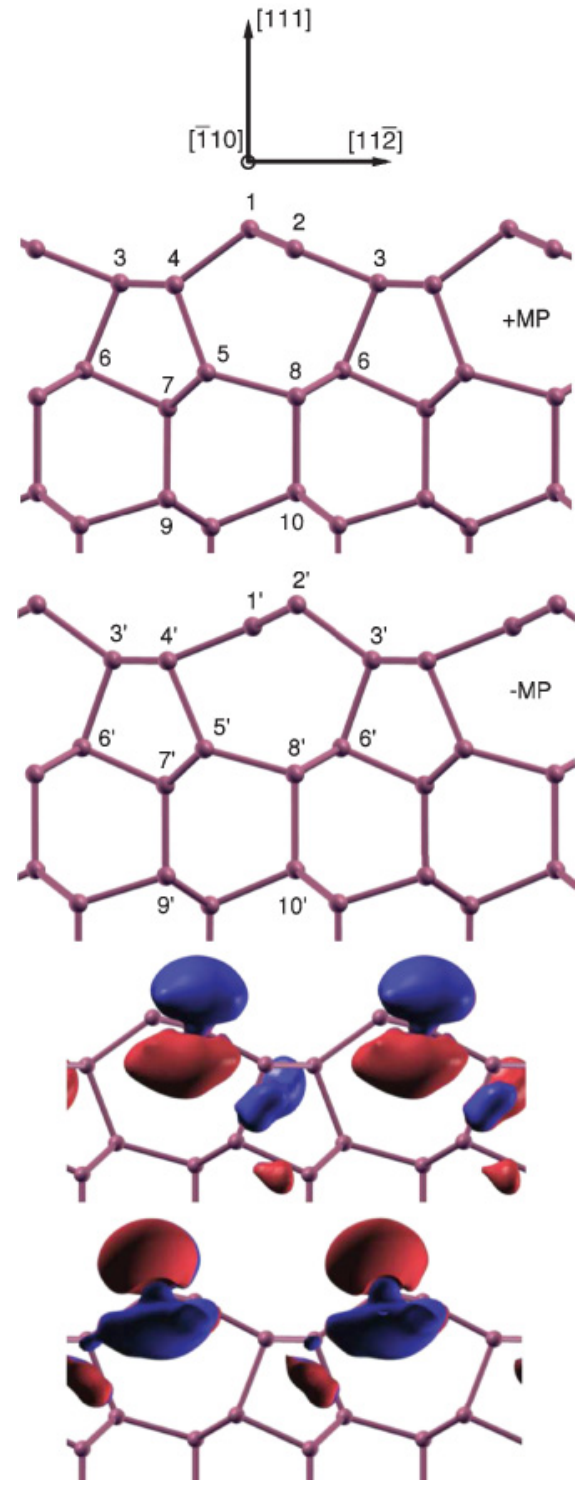

FIG. 1. (Color online) Top panels: Modified Pandey (MP) chain model atomic structures with positive $(+\mathrm{MP})$ and negative $(-\mathrm{MP})$ tilts, viewed along the [110] (chain) direction. Lower panels: Vacant (upper) and occupied (lower) surface states of the +MP structure at the $\bar{J}$ point of the surface Brillouin zone.

Appendix) contains Fock exchange with a weight chosen to match the predicted and experimental values of the static, long-wavelength dielectric constant of $\mathrm{Si}$. This also results in the $E_{2}$ peak of the Si bulk dielectric function coinciding with the experimental position. The hybrid DFT method allows structures, RAS spectra, phonon energies, and the phonon contribution to the conductivity for both the $+\mathrm{MP}$ and -MP variants of the modified Pandey $\pi$-bonded chain structure to be obtained efficiently. Surface structures, surfacestate electronic structures, results of RAS calculations, and phonon modes and surface conductivities are given in the following section and are followed by a brief discussion and conclusions.

\section{RESULTS}

\section{A. Surface structure}

Views of the \pm MP structures along the [1110] and [111] directions are shown in Figs. 1 and 2. Figure 2 also shows the Brillouin zone for the $2 \times 1$ surface unit cell. Previous total energy minimization calculations on these surfaces which compared $\pm \mathrm{MP}$ surfaces found the -MP structure to be more stable by $2.7 \mathrm{meV} /$ surface atom $^{8}$ or $6 \mathrm{meV} /$ surface atom, ${ }^{5}$ while we find that the $+\mathrm{MP}$ structure is more stable by $4 \mathrm{meV} /$ surface atom using hybrid DFT. The most recent analysis of LEED data for the $\mathrm{Si}(111)-2 \times 1$ surface $^{4}$ considered the $+\mathrm{MP}$ structure and mixtures of $\pm \mathrm{MP}$ structures, but did not consider the - MP structure separately. A recent DFT and $G W$ study of these surfaces ${ }^{23}$ found both \pm MP structures to have a small total energy difference, but did not quote a value for the total energy difference nor specify the ground state.

In this work, total energies of slabs containing 12 bilayers and $40 \mathrm{Si}$ ions were minimized using the CRYSTAL code ${ }^{25}$ and a basis set described in the Appendix. Both surfaces of each slab had either + MP or - MP structures. Relaxed lattice parameters for the $2 \times 1$ slab are 7.719 and $3.878 \AA$, which compare to the bulk lattice $1 \times 1$ distance, $3.839 \AA$; the slab lattice parameters are therefore overestimated by hybrid DFT by 1.0 and $0.57 \%$, respectively. Bond lengths derived from the relaxed \pm MP structures are given in Table I, where they are compared to bond lengths derived from the most recent analysis of LEED data for this surface ${ }^{4}$ and a previous DFT calculation. ${ }^{8}$

When bond lengths for the +MP structure in Table I from the DFT calculation, hybrid DFT calculation, and LEED analysis are compared, most bond lengths from LEED lie between the DFT and hybrid DFT values. This is to be expected since hybrid DFT and DFT, respectively, generally overestimate or underestimate experimentally determined bond lengths by a several hundredths of an $\AA$. Three bond lengths derived from LEED data in Table I do not fall between the DFT and hybrid DFT bond lengths. These are the $1-4,5-8$, and $6-7$ bond lengths. Bond 1-4 is the back bond between the outermost atom in the $\pi$ chain and the atom in the layer beneath. $\mathrm{Xu}$ et $a l .{ }^{4}$ find it to be $2.313 \AA$, while a bond length of $2.37 \AA$ seems more likely from the average of bond lengths from
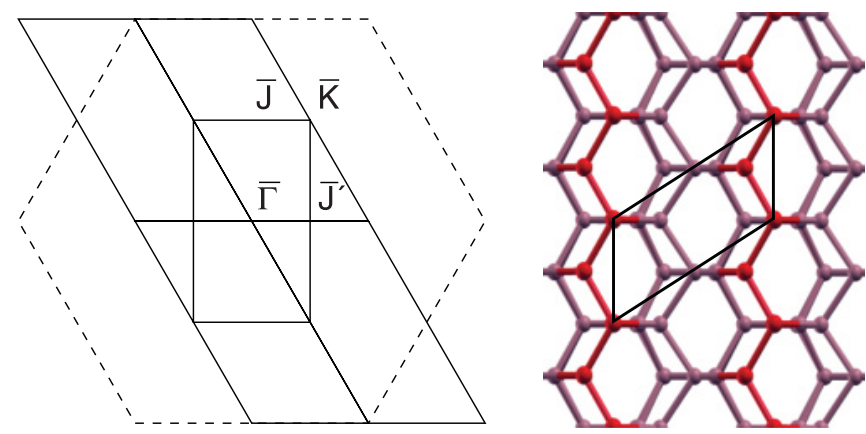

FIG. 2. (Color online) Left: Four reciprocal space unit cells and surface Brillouin zone for the $2 \times 1$ superlattice of the MP chain model (solid lines). The dotted lines connect points of the $1 \times 1$ surface reciprocal lattice. Right: Plan view of the $2 \times 1$ superlattice of the MP chain model real-space unit cell. 
TABLE I. Surface bond lengths in Å in MP structures from LDA DFT, hybrid DFT, and LEED.

\begin{tabular}{lccccc}
\hline \hline Atom Pair & $-\mathrm{MP}^{\mathrm{a}}$ & $+\mathrm{MP}^{\mathrm{a}}$ & $-\mathrm{MP}^{\mathrm{b}}$ & $+\mathrm{MP}^{\mathrm{b}}$ & LEED $+\mathrm{MP}^{\mathrm{c}}$ \\
\hline $1-2$ & 2.25 & 2.25 & 2.316 & 2.312 & 2.271 \\
$1-4$ & 2.30 & 2.34 & 2.358 & 2.414 & 2.313 \\
$2-3$ & 2.34 & 2.30 & 2.411 & 2.354 & 2.347 \\
$3-4$ & 2.34 & 2.34 & 2.398 & 2.401 & 2.384 \\
$4-5$ & 2.41 & 2.37 & 2.466 & 2.422 & 2.371 \\
$3-6$ & 2.35 & 2.39 & 2.395 & 2.441 & 2.423 \\
$5-7$ & 2.34 & 2.33 & 2.374 & 2.371 & 2.359 \\
$5-8$ & 2.34 & 2.36 & 2.416 & 2.405 & 2.277 \\
$6-7$ & 2.33 & 2.34 & 2.354 & 2.368 & 2.393 \\
$6-8$ & 2.34 & 2.34 & 2.381 & 2.384 & 2.386 \\
$7-9$ & 2.30 & 2.30 & 2.346 & 2.343 & \\
$8-10$ & 2.40 & 2.39 & 2.465 & 2.465 & \\
Bulk & & & $2.371^{\mathrm{d}}$ & & 2.352 \\
\hline \hline
\end{tabular}

${ }^{\mathrm{a}}$ LDA DFT Ref. 8.

${ }^{\mathrm{b}}$ Hybrid DFT (this work).

${ }^{c}$ Reference 4.

${ }^{\mathrm{d}}$ Hybrid DFT calculation on bulk cell using same basis set (this work).

total energy calculations (Table I). Bond 5-8 is a bulklike bond in the layer below the $\pi$ chain. $\mathrm{Xu}$ et al. find it to be $2.277 \AA$ while a bond length of $2.38 \AA$ is more likely. $\mathrm{Xu}$ et al. find the adjacent bulklike bond 6-7 to be $2.39 \AA$, while a bond length of $2.35 \AA$ is predicted. Previous studies of the structure of the $\mathrm{Si}(111)-2 \times 1$ surface have reported the extent of buckling, i.e., the relative vertical displacement of atoms 1 and 2, which is known as the $b_{1}$ parameter. $\mathrm{Xu}$ et al. find it to be $0.51 \AA$ for the $+\mathrm{MP}$ structure, while our hybrid DFT calculations find $b_{1}=0.513 \AA$ for $+\mathrm{MP}$ and $0.559 \AA$ for the $-\mathrm{MP}$ structure. Zitzlsperger et al. find $b_{1}=0.4 \AA$ for the $+\mathrm{MP}$ and $0.50 \AA$ for the -MP structure, ${ }^{8}$ while Bussetti et al. find $b_{1}=0.53 \AA$ for the $+\mathrm{MP}$ and 0.59 $\AA$ for the - MP structure. ${ }^{23}$ These values are in reasonable agreement with LEED data for the +MP structure, and DFT and hybrid DFT calculations agree that buckling in the -MP structure is larger.

\section{B. Electronic structure}

There are two Si atoms per surface unit cell in the $\pi$ chains of the \pm MP structures and each has a dangling Si $3 p$ state oriented roughly perpendicular to the chain. These dangling $p$ states contain two electrons and combine to form two surface states (Figs. 1 and 3); close to the $\bar{J}$ and $\bar{K}$ points of the surface Brillouin zone, the occupied state is strongly localized on the outermost atom in the $\pi$ chains of both MP structures while the vacant state is strongly localized on the inner atom of the $\pi$ chains. Around the $\bar{\Gamma}$ and $\overline{J^{\prime}}$ points of the surface Brillouin zone the surface states mix with near-surface bulk states.

Dispersion of filled ${ }^{26}$ and vacant ${ }^{22}$ surface-state bands in the Si(111)- $2 \times 1$ surface has been mapped by ARPES and IPES. In heavily doped $n$-type samples an additional peak is observed in ARPES at the $\bar{J}$ point of the surface Brillouin zone. ${ }^{20,23}$ This was attributed to filling of the vacant surface state at its minimum energy point by extra $n$-type carriers introduced by doping.

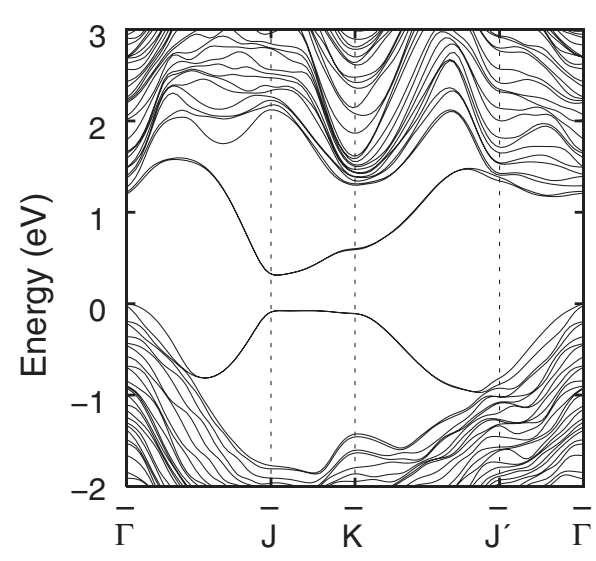

FIG. 3. Electronic band structure for $+\mathrm{MP}$ structure.

The electronic band structure from hybrid DFT calculations for the +MP structure is shown in Fig. 3. While the band structures of either MP structure are similar, there are subtle differences. Relative positions of surface-state energies from hybrid DFT calculations in both structures are given in Table II. All energies are referred to the valence band maximum (VBM) energy at the $\bar{\Gamma}$ point of the + MP structure. The indirect band gap $(\bar{\Gamma}-\bar{J})$ for the +MP structure is $316 \mathrm{meV}$, while the minimum direct gap at $\bar{J}$ is $398 \mathrm{meV}$. In the $-\mathrm{MP}$ structure the indirect $\bar{\Gamma}-\bar{J}$ gap is $282 \mathrm{meV}$, while the minimum direct gap at $\bar{J}$ is $310 \mathrm{meV}$. The conduction band minimum (CBM) at $\bar{J}$ for the -MP structure lies $247 \mathrm{meV}$ above the VBM of the +MP structure, while the CBM for the +MP structure lies $69 \mathrm{meV}$ higher in energy. While differences in single-particle energy eigenvalues cannot be exactly equated with total energy differences, in this case there is a large difference in single-particle positions of the CBM (69 meV), which greatly exceeds the difference in the calculated total energy of $8 \mathrm{meV}$, so it is likely that the presence of $n$-type carriers at the surface will cause a transition to the -MP structure from the +MP structure. This is in agreement with the conclusion of recent work by Bussetti et al. ${ }^{23}$ The difference in single-particle positions of the CBM for the \pm structures determined by STM was $0.07 \mathrm{eV},{ }^{23}$ in very good agreement with the value of $69 \mathrm{meV}$ from the hybrid DFT calculation.

The presence of strong electron-electron effects in this surface was noted in Sec. I. $G W$ calculations ${ }^{7,23}$ of surfacestate energies and BSE calculations of the optical band gap, ${ }^{10,27}$ which include excitonic electron-hole effects, have been reported previously. HOMO-LUMO gaps from STM and ARPES experiments and $G W$ calculations are given in the first two columns of Table III. The excitonic binding energy

TABLE II. Positions of surface states in meV relative to the $\bar{\Gamma}$ point valence band maximum in the + MP structure.

\begin{tabular}{lrr}
\hline \hline$k$ point & + MP & + MP \\
\hline $\bar{\Gamma}(\mathrm{VBM})$ & 0 & -35 \\
$\bar{J}$ & -82 & -63 \\
$\bar{K}$ & -114 & -103 \\
$\bar{J}(\mathrm{CBM})$ & 316 & 247 \\
\hline \hline
\end{tabular}


TABLE III. HOMO-LUMO (HL) gap in \pm MP structures and optical excitation energies in the +MP structure in $\mathrm{VV}$ from STM and ARPES experiment and hybrid DFT, $G W$, and BSE calculations.

\begin{tabular}{lccc}
\hline \hline & HL Gap +MP & HL Gap -MP & Optical Gap +MP \\
\hline STM $^{\mathrm{a}}$ & 0.83 & 0.47 & \\
ARPES $^{\mathrm{b}}$ & & 0.45 & \\
Hybrid DFT $^{\mathrm{c}}$ & 0.40 & 0.31 & 0.40 \\
$G W^{\mathrm{d}}$ & 0.75 & & 0.45 \\
$G W^{\mathrm{e}}$ & 0.62 & & 0.47 \\
$G W^{\mathrm{f}}$ & 0.69 & & 0.43 \\
$G W^{\mathrm{g}}$ & 0.79 & & \\
\hline \hline
\end{tabular}

${ }^{\mathrm{a}}$ STM data, Ref. 23.

${ }^{\mathrm{b}}$ ARPES data for $n$-type surface with both surface states occupied at $\bar{J}$ point, Ref. 23.

${ }^{\mathrm{c}}$ This work.

${ }^{\mathrm{d}}$ Calculation in tight-binding basis using fitted HL gap, Ref. 27.

ePlasmon pole approximation applied to diagonal elements of inverse dielectric matrix, Ref. 7.

${ }^{\mathrm{f}}$ Generalized plasmon pole approximation, Ref. 10.

${ }^{g}$ DFT calculation with conduction bands shifted upward by $0.5 \mathrm{eV}$ based on self-energy estimates at $4 k$ points, Ref. 23.

is the difference in the single particle HOMO-LUMO gap and the optical gap (third column of Table III) and ranges from 0.15 to $0.30 \mathrm{eV}$ in BSE calculations for this surface. The $G W$ calculations which are cited in Table III use dielectric functions with varying degrees of approximation, but are generally in agreement with experimental estimates of the HOMO-LUMO gap for the +MP structure, $0.83 \mathrm{eV}$, from STM. $^{23}$

Any theoretical method which predicts particle or hole energies correctly will overestimate the optical transition energy by the excitonic binding energy, if electron-hole interactions in the excited state are omitted. In the hybrid DFT method used here, the functional used has been adjusted so that the bulk silicon static dielectric function and absorption maximum in the $\epsilon_{2}(\omega)$ spectrum agree with experiment when no electronhole effects are included in the optical spectrum calculation. This is achieved by varying the relative amount of Fock exchange in the functional (see Appendix) as the predicted band gap increases approximately linearly with the relative amount of Fock exchange. A similar approach is commonly adopted when DFT calculations are used to compute optical spectra without inclusion of many-body effects-conduction bands are simply shifted upward until optimal agreement with experiment is achieved. ${ }^{28}$ In the present work the shift occurs because Fock exchange has been included in the exchange correlation functional and wave functions and energy eigenvalues are obtained self-consistently.

The hybrid DFT HOMO-LUMO gap for the +MP structure is $0.40 \mathrm{eV}$, smaller than the gap from $G W$ calculations cited in Table III by approximately the excitonic binding energy. The onset of optical absorption in the $\mathrm{Si}(111)-2 \times 1$ surface occurs between 0.3 and $0.4 \mathrm{eV}$, according to differential reflectivity ${ }^{17}$ and RAS experiments. ${ }^{29}$ The optical gaps predicted by our hybrid DFT calculations and BSE calculations range from 0.40 to $0.47 \mathrm{eV}$ (Table III).

\section{Reflectance anisotropy}

As mentioned above, the occurrence of filled and vacant surface states localized on adjacent $\mathrm{Si}$ atoms in $\pi$ chains of the \pm MP structures results in large charge transfers between filled and vacant $3 p$ dangling bond states and large optical absorption cross sections. Optical transitions at surfaces can be observed using the RAS technique in which the reflectance of light incident normal to the surface is measured with the optical electric vector parallel to one or the other symmetry axis in the surface.$^{30}$ For the Si(111)- $2 \times 1$ surface these directions are parallel or perpendicular to the $\pi$ chains. The RAS signal is

$$
\frac{\Delta r}{r}=2 \frac{r_{x}-r_{y}}{r_{x}+r_{y}}
$$

where $r_{x}$ and $r_{y}$ are complex reflectivity amplitudes measured with the electric vector parallel to the $x(=[11 \overline{2}])$ and $y$ $(=[\overline{1} 10])$ directions. We use the McIntyre-Aspnes 3-layer model $^{31}$ to calculate the reflectivity of a surface from the dielectric function for a slab of finite thickness, in this case just over $30 \AA$ thick. The change $\Delta R$ in average reflectivity $R$, induced by a surface layer with a nonzero, anisotropic, surface excess susceptibility $\Delta \chi_{x s}$ relative to the average reflectivity, is given by

$$
\frac{\Delta R}{R}=2 \operatorname{Re} \frac{\Delta r}{r}=4 k d \operatorname{Im} \frac{\left(\Delta \chi_{x s}^{i} \chi_{b}^{r}-\Delta \chi_{x s}^{r} \chi_{b}^{i}\right)}{\left|\chi_{b}\right|^{2}},
$$

where

$$
\Delta \chi_{x s}(\omega)=\chi_{x s, x}(\omega)-\chi_{x s, y}(\omega),
$$

the superscripts $r$ and $i$ indicate real and imaginary parts, $k$ is the wave vector magnitude of the incident light, and $d$ is the surface layer thickness. The frequency-dependent surface excess susceptibility ${ }^{32}$ is defined by

$$
\chi_{x s, i}(\omega)=\chi_{s, i}(\omega)-\chi_{b}(\omega),
$$

where $\chi_{s, i}$ is the surface susceptibility in direction $i$, and $\chi_{b}$ is the bulk susceptibility. Surface and bulk susceptibilities are calculated using the single-particle susceptibility expression

$$
\chi_{i}(\omega)=\frac{2 e^{2}}{m^{2} \epsilon_{o} \Omega \omega^{2}} \sum_{n n^{\prime} \mathbf{k}} \frac{\left[f_{o}\left(E_{n \mathbf{k}}\right)-f_{o}\left(E_{n^{\prime} \mathbf{k}}\right)\right]\left|p_{n n^{\prime} \mathbf{k}}^{i}\right|^{2}}{\left(E_{n n^{\prime} \mathbf{k}}-E-i \delta\right)},
$$

where $f_{o}$ is a Fermi occupation factor, $p_{n n^{\prime} \mathbf{k}}^{i}$ is a momentum matrix element connecting states $n \mathbf{k}$ and $n^{\prime} \mathbf{k}$ in the presence of a field along direction $i, E_{n n^{\prime} \mathbf{k}}$ is the corresponding transition energy, and $m, e$, and $\Omega$ are the electron mass, charge, and unit cell volume. Surface and bulk susceptibilities were calculated using the EXCITON code $^{24}$ using a $16 \times 32$ grid of $k$ points in the surface Brillouin zone and a $24 \times 24 \times 24$ grid in the bulk Brillouin zone. Integration over the Brillouin zone was performed using an interpolation method. ${ }^{33}$

RAS spectra calculated for the \pm MP structures are compared to a RAS spectrum from experiment ${ }^{29}$ in Fig. 4. No scaling has been applied to the spectra from hybrid DFT calculations. The RAS spectrum below $1 \mathrm{eV}$ is a single, asymmetric peak with a sharp leading edge. The experimental peak position is $0.46 \mathrm{eV}$ while the predicted peak positions for the $+\mathrm{MP}$ and $-\mathrm{MP}$ structures are 0.56 and $0.49 \mathrm{eV}$, respectively. The 


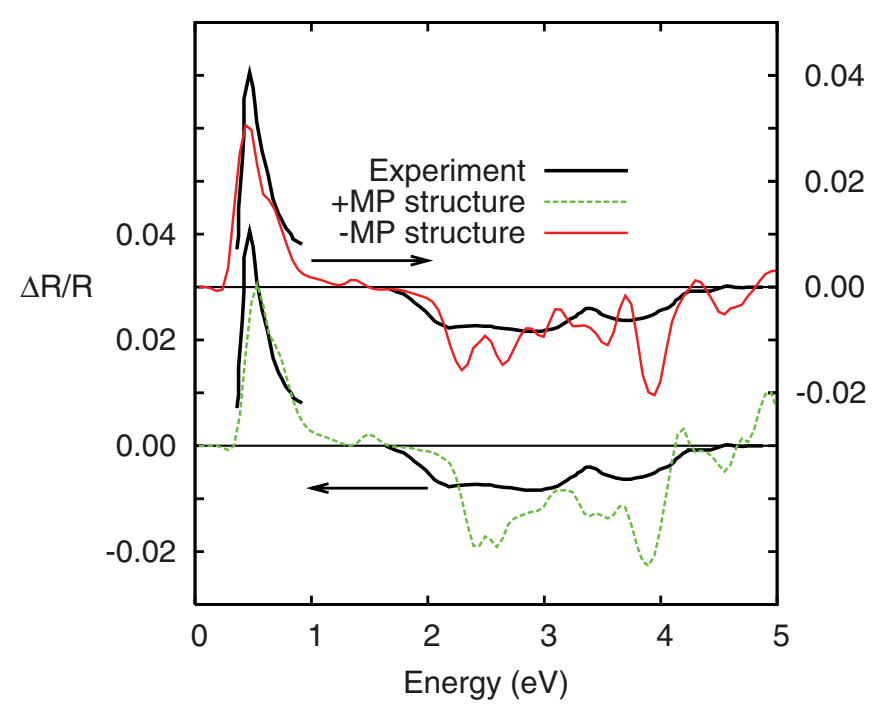

FIG. 4. (Color online) Reflectance anisotropy spectra for $\pm \mathrm{MP}$ structures compared to experimental data. Experimental data are redrawn from Ref. 29 and were collected in two spectral regions.

linewidth for either MP structure is greater than the experimental linewidth. Rohlfing and Louie found a quasiparticle band gap of $0.69 \mathrm{eV}^{10}$ in a $G W$ calculation for this surface and an exciton binding energy of $0.26 \mathrm{eV}$ in a BSE calculation.

The optical response of this surface is highly anisotropic, being very large parallel to the $\pi$ chains and very small perpendicular to the chains in the infrared [compared to, for example, the $\mathrm{Si}(100)-2 \times 1$ surface $^{28}$ ]. In the visible region (between 2 and $4 \mathrm{eV}$ ) the imaginary part of the surface susceptibility perpendicular to the $\pi$ chains is greater than that parallel to the chains. Figure 5 shows a surface wave vector dependent gray-scale plot of the modulus squared of the momentum matrix element for transitions between the occupied and vacant surface states for the -MP structure when the electric vector of the incident radiation is parallel or perpendicular to the $\pi$ chains. Transitions between states other than the two surface states were excluded when generating Fig. 5. When the electric vector is parallel to the chains, this matrix element is maximal at the $\bar{J}$ point and has large amplitude only along the $\bar{K}-\bar{J}-\bar{K}$ line in the Brillouin zone. When the electric vector is perpendicular to the chains, the matrix element vanishes in this region of reciprocal space and instead is large close to the $\overline{J^{\prime}}$ points.

The surface susceptibility depends on both the momentum matrix element and, at resonance, the inverse square of the transition energy [Eq. (5)]. Transition energies for the occupied and vacant surface states in the -MP structure, as a function of position in the surface Brillouin zone, are shown in Fig. 6. Figures 5 and 6 show that predominance of the response along the $\pi$ chains in the infrared depends on both optical matrix elements and transition energies. When the electric vector is parallel to the $\pi$ chains, the matrix element at $\bar{J}$ is large and the corresponding transition energy is small (around $0.3 \mathrm{eV}$ ); on the other hand, when the electric vector is perpendicular to the $\pi$ chains, the matrix element is large close to $\overline{J^{\prime}}$ but the transition energy is large (over $2.0 \mathrm{eV}$ ), leading to a large

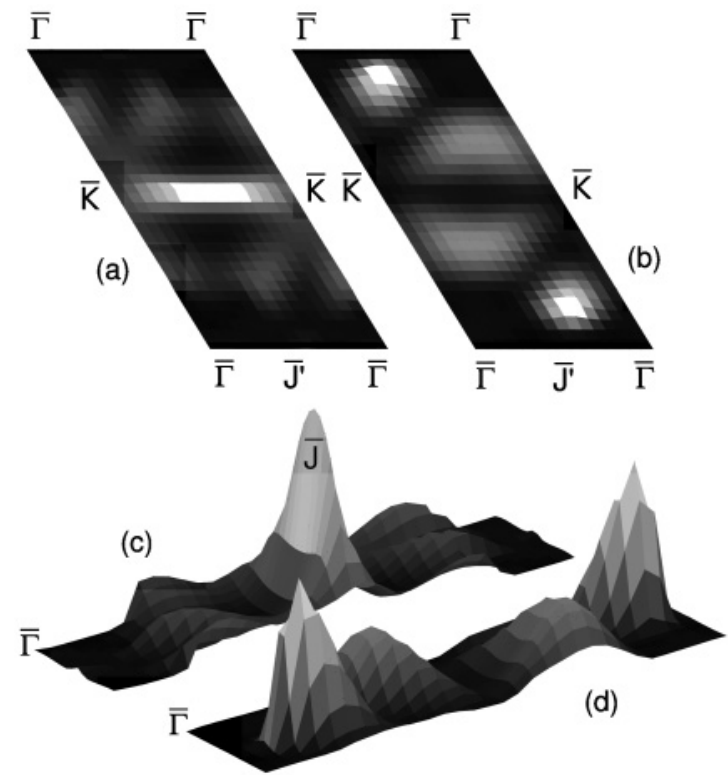

FIG. 5. Modulus squared of the transition matrix element, $p_{n n^{\prime} \mathbf{k}}^{i}$, between occupied and vacant surface states over one unit cell in reciprocal space for the $-\mathrm{MP}$ structure. (a), (c) Field parallel to [1110]. (b), (d) Field parallel to [11 $\overline{2}]$.

difference $(\sim 300)$ in the optical response magnitude, parallel or perpendicular to the $\pi$ chains.

\section{Phonons and IR conductivity}

HREELS measurements ${ }^{12,13}$ and calculations ${ }^{8,14,16}$ of phonons at the $\operatorname{Si}(111)-2 \times 1$ surface show that specular,
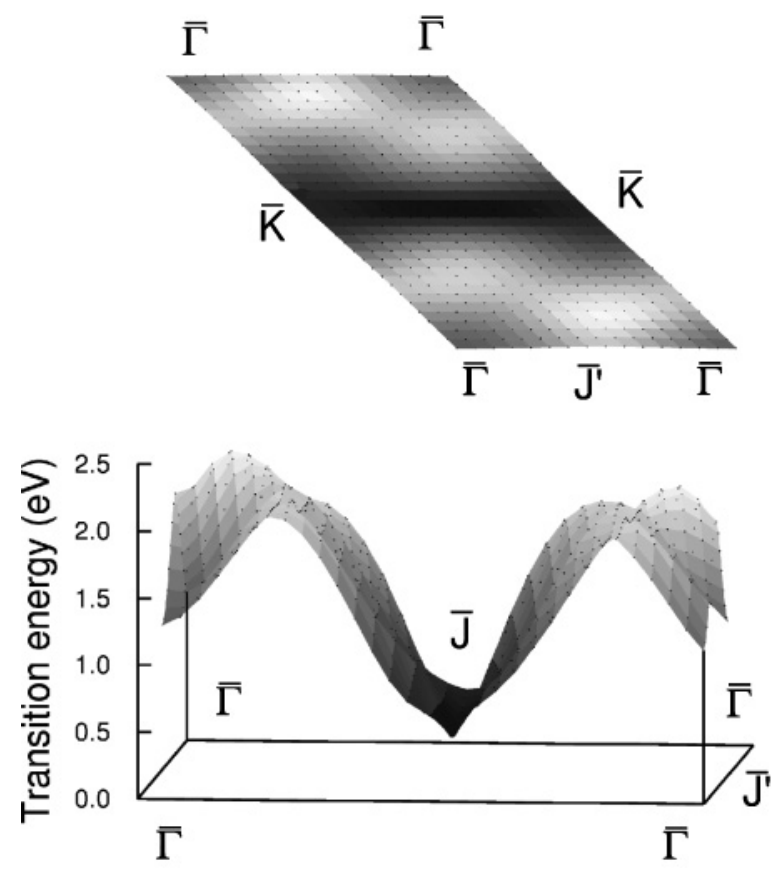

FIG. 6. Optical transition energy between occupied and vacant surface states over one unit cell in reciprocal space for the -MP structure. Darker areas in the upper panel correspond to lower transition energies. 
TABLE IV. Born effective charges in units of the electronic charge, $e$, for surface atoms in the $\pm \mathrm{MP}$ structures.

\begin{tabular}{lrrrr}
\hline \hline \multicolumn{2}{c}{+ MP Structure } & & \multicolumn{2}{c}{-MP Structure } \\
\cline { 5 - 6 } Atom & Born Charge & & Atom & Born Charge \\
\cline { 5 - 6 } 1 & -2.84 & & $1^{\prime}$ & 3.48 \\
2 & 2.88 & & $2^{\prime}$ & -3.42 \\
3 & -0.07 & & $3^{\prime}$ & 0.12 \\
4 & 0.20 & & $4^{\prime}$ & -0.02 \\
5 & -0.32 & & $5^{\prime}$ & -0.06 \\
6 & 0.09 & & $6^{\prime}$ & -0.24 \\
7 & -0.09 & & $7^{\prime}$ & -0.04 \\
8 & 0.16 & & $8^{\prime}$ & 0.25 \\
\hline \hline
\end{tabular}

inelastic scattering of low-energy electrons with $k_{\|}$along the [110] direction is dominated by a phonon loss at $55^{12}$ or $57.5 \mathrm{meV} .^{13}$ The anomalously strong scattering by a phonon polarized parallel to the $\pi$ chains arises because of large electron transfer between $\mathrm{Si}$ atoms in $\pi$ chains, when they move along the [110] direction. This charge transfer can be quantified using the concept of the Born effective charge tensor. The tensor $Z^{*}$ for an atom in its local, Cartesian coordinate system is

$$
Z_{\alpha, i j}^{*}=\frac{\partial \mu_{i}}{\partial u_{\alpha j}},
$$

where $\mu_{i}$ is the dipole moment induced in direction $i$ when atom $\alpha$ undergoes displacement $u_{j}$ along $j$. Born charges are defined to be one-third of the trace of the Born charge tensor. Charges for atoms labeled 1 to 8 and $1^{\prime}$ to $8^{\prime}$ for the $\pm \mathrm{MP}$ structures in Fig. 1 are given in Table IV. There is a change in sign of the Born charges of the $\pi$-chain atoms upon switching between \pm MP structures because changing structure switches the location of occupied and vacant surface states on these atoms. The charges for the two $\mathrm{Si}$ atoms in the $\pi$ chains are anomalously large and may be compared with the value for bulk $\mathrm{Si},-9 \times 10^{-6} e$, obtained using the same basis set. The large values of the Born charges for $\pi$-chain atoms, compared to charges for atoms in layers beneath, means that polarization induced by phonon excitation is determined mainly by the relative motions of the $\pi$-chain atoms.

The phonon contribution to the (diagonal) surface conductivity tensor in $\mathrm{S}$ per square is

$$
\sigma_{i}(\omega)=\frac{1}{A} \sum_{p} \frac{\gamma \omega^{2} Z_{p, i} Z_{p, i}}{\omega_{p}^{2}-\omega^{2}-i \omega \gamma},
$$

where $Z_{p, i}$ is a diagonal element of the Born charge tensor of the $p$ th phonon coordinate, $\omega_{p}$ is the corresponding phonon frequency, and $\gamma$ is a line broadening parameter, chosen here to be $1 \mathrm{meV}$. $A$ is the area of the surface unit cell. The Born charge in a phonon coordinate is obtained by contracting Born charge tensors in local atomic coordinates for each ion with the (dimensionless) displacements of the ions in the phonon mode. The transformation between Born charges in local, atom-centered coordinates and phonon coordinates is

$$
Z_{p, i}=\sum_{\alpha, j} \frac{t_{p, \alpha j} Z_{\alpha, i j}^{*}}{\sqrt{M_{\alpha}}}
$$

TABLE V. Mode energies (meV) and Born effective charges in phonon coordinates, $Z_{p, i}$, in units of the electronic charge, $e$, for phonons in the \pm MP structures.

\begin{tabular}{lcrr}
\hline \hline Mode Energy & $Z_{x}$ & \multicolumn{1}{c}{$Z_{y}$} & \multicolumn{1}{c}{$Z_{z}$} \\
& + MP Structure & & \\
\hline 17.2 & 0.01 & -0.27 & -0.01 \\
20.8 & 0.27 & 0.03 & 0.01 \\
47.6 & 0.18 & 0.01 & -0.02 \\
50.4 & 0.04 & -1.90 & -0.06 \\
55.9 & 0.01 & -0.61 & -0.01 \\
57.4 & 0.01 & -0.47 & -0.06 \\
58.8 & 0.02 & -0.92 & -0.02 \\
58.9 & -0.10 & -0.03 & -0.01 \\
& - MP Structure & & \\
18.0 & 0.00 & -0.32 & 0.00 \\
20.8 & 0.23 & 0.03 & 0.00 \\
47.3 & -0.12 & 0.18 & 0.00 \\
47.7 & 0.02 & -2.49 & 0.00 \\
55.9 & 0.00 & -0.55 & 0.00 \\
57.3 & -0.00 & 0.49 & 0.00 \\
58.4 & -0.01 & 0.63 & 0.00 \\
60.2 & 0.10 & -0.01 & 0.00 \\
\hline \hline
\end{tabular}

where $M_{\alpha}$ is the mass of the $\alpha$ th ion and $t_{p}$ is the $p$ th phonon eigenvector. Phonon frequencies and Born charges are given in Table V. Born charge tensor components parallel to the $\pi$ chains, $Z_{y}$, are as much as an order of magnitude larger than those in other directions, and this accounts for the factor of 100 variation in the coordinate axis scales in Fig. 7, allowing for the quadratic dependence of the surface conductivity on the Born charges.

\section{DISCUSSION AND CONCLUSIONS}

The hybrid DFT approach is an efficient way of calculating surface structure, electronic structure, optical RAS spectra, and the phonon contribution to the surface conductivity. Hybrid DFT Hamiltonians contain weighted Fock exchange and this increases the surface and bulk band gaps compared to, say, an LDA DFT Hamiltonian. The weight of Fock exchange in the Hamiltonian in this work (see Appendix) was chosen so that the real part of the static dielectric function agrees with the experimental value. An important consequence is that the main $E_{2}$ peak of the bulk Si dielectric function appears at the correct energy, providing evidence that this approach delivers a useful correction to single-particle interband transition energies, when compared to transition energies derived from LDA DFT Hamiltonians. This approach does not, however, incorporate excitonic effects. A major advantage of a local orbital basis is that all methods used (energy minimization, phonon mode calculation, electronic structure, and optical response) have the same hybrid DFT Hamiltonian; this is possible because all of these calculations are relatively fast, whereas $G W$ and BSE methods for computing the optical spectrum are significantly slower. In particular, this approach is suited for calculating the optical response of more complex surface and interface structures with large unit cells. 


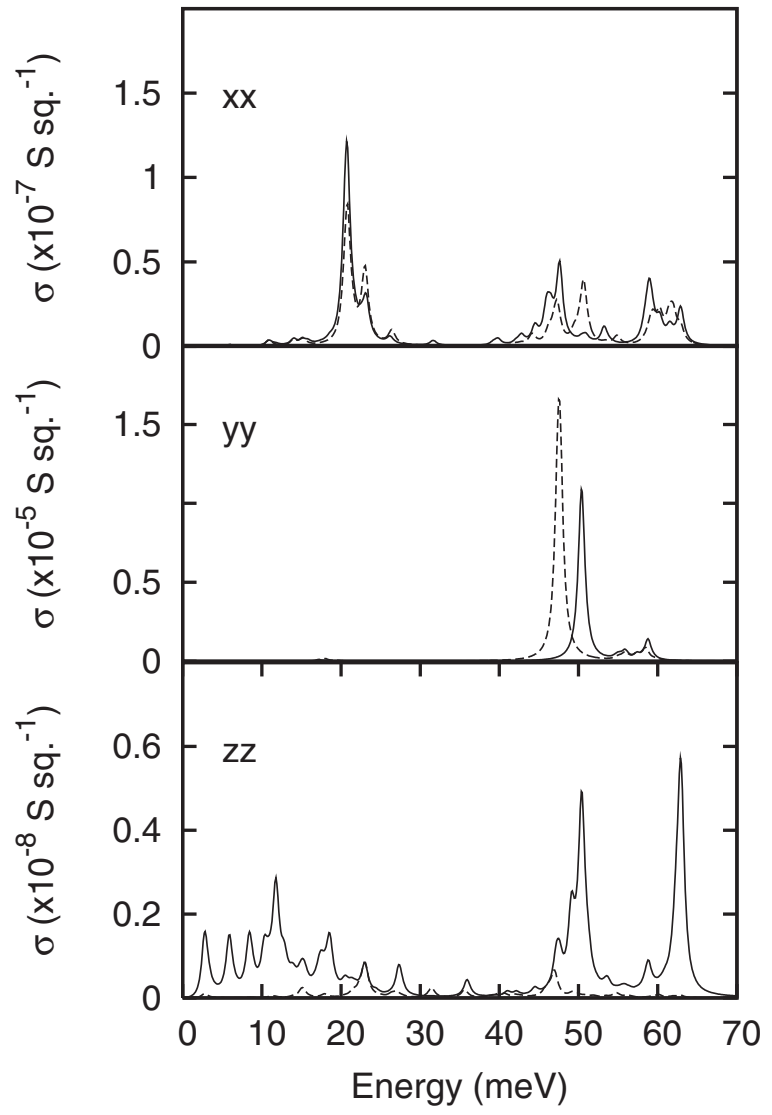

FIG. 7. Surface conductivity tensor elements for \pm MP surfaces in $\mathrm{S}$ sq. ${ }^{-1}$. $+\mathrm{MP}$, solid lines; -MP, dotted lines. Note differences in scales of axes. $x=[11 \overline{2}], y=[\overline{1} 10], z=[111]$.

It has recently been shown that both \pm MP structures exist at the $\mathrm{Si}(111)-2 \times 1$ surface and that $n$-type carriers in a heavily doped surface favor formation of the -MP structure. ${ }^{23}$ According to our hybrid DFT calculations, the total energy of the +MP structure is $4 \mathrm{meV}$ /surface atom lower in energy than the -MP structure. In this work we found that positions of surface states associated with $\pi$ chains in the \pm MP structures are such that the CBM of the -MP structure lies $69 \mathrm{meV}$ lower than the CBM of the +MP structure. It is therefore likely that the addition of $n$-type carriers to the $\operatorname{Si}(111)-2 \times 1$ surface does indeed favor the - MP structure. Our hybrid DFT calculations predict that the + MP structure is lower in energy, apparently in agreement with experiment for the undoped $\mathrm{Si}(111)-2 \times 1$ surface, ${ }^{23}$ whereas previous DFT calculations ${ }^{5,8}$ have found the -MP structure to be lower in energy.

Bond lengths are compared for $11 \mathrm{Si}-\mathrm{Si}$ bonds at the Si(111)- $2 \times 1$ surface derived from hybrid (this work) and LDA $^{8}$ DFT calculations and a reanalaysis of LEED data. ${ }^{4}$ In most cases, bond lengths from LEED data lie between those predicted by the two DFT approaches. Since hybrid and LDA DFT methods generally over or underestimate bond lengths, respectively, it is likely that in the few cases where atomic positions from LEED analysis do not lie between those predicted by LDA and hybrid DFT methods, they need to be modified. Mostly, however, there is good agreement between bond lengths from LEED analysis and the mean values of bond lengths predicted by LDA and hybrid DFT methods.
The optical RAS spectrum for the \pm MP structures have maxima at 0.56 and $0.49 \mathrm{eV}$, respectively, just above the experimental peak position, $0.46 \mathrm{eV}^{29}$ The lower value for the peak position of the -MP structure reflects its smaller, direct, single-particle band gap (310 meV, cf. $398 \mathrm{meV}$ for the $+\mathrm{MP}$ structure). These values are somewhat less than the quasiparticle gap from a $G W$ calculations on the $+\mathrm{MP}$ surface, $, 10,23,27$ which range between 0.62 and $0.79 \mathrm{eV}$. BSE calculations ${ }^{7,10,27}$ found exciton binding energies in the range 0.15 to $0.30 \mathrm{eV}$. Thus the surface-state gap predicted by the hybrid DFT method used here is approximately $0.3 \mathrm{eV}$ less than that predicted by $G W$ calculations. Since excitonic electron-hole binding is omitted from our calculations, the optical gap is simply the difference in single-particle energy levels. A plot of the optical transition matrix elements for transitions between occupied and vacant surface states shows that the optical anisotropy depends on both matrix elements and optical transition energies. Parallel to $\pi$-bonded chains the matrix element is large where the optical transition energy is small; perpendicular to the chains the matrix element is large only where the optical transition energy is also large.

The phonon contribution to the conductivity of the $\mathrm{Si}(111)$ $2 \times 1$ surface was calculated using Born effective charges and phonon mode frequencies. The conductivity has previously been calculated using phonon modes from a tight-binding calculation. ${ }^{14}$ Our calculations are in fairly good agreement with those earlier calculations, although we find the magnitude of the Born effective charges of the $\pi$-chain atoms to be even larger than those reported previously $(-2.84$ and $2.88 e$ for the +MP structure and 3.48 and $-3.42 e$ for the $-\mathrm{MP}$ structure, compared to $\pm 1.5 e^{14}$ ). Experimental values for the energy of the mode with a large HREELS scattering cross section for electrons with $k_{\|}$along the [110] direction are $55^{12}$ and $57.5 \mathrm{meV},{ }^{13}$ about $5 \mathrm{meV}$ higher than the values which we obtain for the +MP $(50.4 \mathrm{meV})$ or -MP $(47.7 \mathrm{meV})$ structures. These phonons are strongly localized on atoms 1 and 2 (or $1^{\prime}$ and $2^{\prime}$ ) in Fig. 1 and are antiphase motions of ions with large Born charges of opposite sign. Dipolar coupling ${ }^{14}$ of the atomic motions and charges in these modes, which has been omitted from our calculations, will lead to strong LO splitting for each of these mode frequencies and an upward shift of the longitudinal frequencies. This is the likely explanation of the discrepancy in the observed ${ }^{12,14}$ and calculated frequencies. The $2.7 \mathrm{meV}$ splitting of the $\pi$-chain localized modes at 50.4 and 47.7 $\mathrm{meV}$ ought to be observable in Raman scattering from these modes when both \pm MP structures are present at a $\mathrm{Si}(111)-2 \times 1$ surface. $^{23}$

In conclusion, the aim of this work has been partly to demonstrate that self-consistent hybrid DFT calculations in a relatively small local orbital basis can be used to predict optical excitation spectra (such as RAS) and surface phonon spectra quite accurately, when the hybrid functional is adjusted so that bulk dielectric properties are predicted as accurately as possible. The computational cost of these calculations is expected to be much lower than equivalent calculations in a plane-wave basis. The approach is therefore suited for application to more complex surface and interface structures with large unit cells. 


\section{ACKNOWLEDGMENTS}

SB is grateful to Trinity College for a postgraduate studentship. Computer time was provided by the Irish Centre for High End Computing and the Trinity Centre for High Performance Computing, which are supported under the Irish National Development Plan. The project was supported by Science Foundation Ireland under Grant No. 09/RFP/MTR2295 and No. 11/RFP/PHY3047.

\section{APPENDIX: DETAILS OF CALCULATIONS}

All self-consistent field calculations were performed using the CRYSTAL program. ${ }^{25}$ Optical cross-section calculations were performed using the EXCITON code, ${ }^{24}$ with wave functions and energy eigenvalues imported from CRYSTAL. The Gaussian orbital basis for $\mathrm{Si}^{34}$ is described in Ref. 35 . The Becke-3 Lee, Yang, and Parr (B3LYP) hybrid density functional $^{36,37}$ contains Hartree-Fock exchange with weight $A$, the local density approximation to exchange, ${ }^{38} E_{x}^{\mathrm{LDA}}$, with weight $1-A$, Becke's gradient corrected exchange functional, ${ }^{39} E_{x}^{\text {Becke, }}$, with weight $B$, the Lee, Yang, and Parr approximation to the correlation functional, ${ }^{40} E_{c}^{\mathrm{LYP}}$, with weight $C$, and the Vosko, Wilks, and Nusair approximation to the electron correlation functional, ${ }^{41} E_{c}^{\mathrm{VWN}}$, with weight $1-C$,

$$
\begin{aligned}
E_{x c}= & (1-A)\left(E_{x}^{\mathrm{LDA}}+B E_{x}^{\mathrm{Becke}}\right)+A E_{x}^{\mathrm{HF}} \\
& +(1-C) E_{c}^{\mathrm{VWN}}+C E_{c}^{\mathrm{LYP}} .
\end{aligned}
$$

The B3LYP functional contains Fock exchange with a weight of 0.2 ; this results in an overestimation of the optical band gap of bulk Si. The weight was reduced to 0.05 , where good agreement with the dielectric function of bulk Si was obtained. The B3LYP hybrid density functional, with $A$ (=0.05), $B$, and $C$ fixed at their standard values, is used throughout this work. The frozen phonon method in CRYSTAL used here is described in Refs. 42 and 43. Born effective charge tensors in CRYSTAL are obtained using a Berry phase method. ${ }^{44}$
${ }^{1}$ K. C. Pandey, Phys. Rev. Lett. 49, 223 (1982).

${ }^{2}$ F. J. Himpsel, P. M. Marcus, R. Tromp, I. P. Batra, M. R. Cook, F. Jona, and H. Liu, Phys. Rev. B 30, 2257 (1984).

${ }^{3}$ H. Sakama, A. Kawazu, and K. Ueda, Phys. Rev. B 34, 1367 (1986). ${ }^{4}$ G. Xu, B. Deng, Z. Yu, S. Y. Tong, M. A. VanHove, F. Jona, and I. Zasada, Phys. Rev. B 70, 045307 (2004).

${ }^{5}$ B. I. Craig and P. V. Smith, Surf. Sci. 225, 225 (1990).

${ }^{6}$ F. Ancilotto, W. Andreoni, A. Selloni, R. Car, and M. Parrinello, Phys. Rev. Lett. 65, 3148 (1990).

${ }^{7}$ J. E. Northrup, M. S. Hybertsen, and S. G. Louie, Phys. Rev. Lett. 66, 500 (1991).

${ }^{8}$ M. Zitzlsperger, R. Honke, P. Pavone, and U. Schröder, Surf. Sci. 377-379, 108 (1997).

${ }^{9}$ F. Ciccacci, S. Selci, G. Chiarotti, and P. Chiaradia, Phys. Rev. Lett. 56, 2411 (1986).

${ }^{10}$ M. Rohlfing and S. G. Louie, Phys. Rev. Lett. 83, 856 (1999).

${ }^{11}$ G. Bussetti, C. Goletti, P. Chiaradia, and G. Chiarotti, Phys. Rev. B 72, 153316 (2005).

${ }^{12}$ H. Ibach, Phys. Rev. Lett. 27, 253 (1971).

${ }^{13}$ N. J. DiNardo, W. A. Thompson, A. J. Schell-Sorokin, and J. E. Demuth, Phys. Rev. B 34, 3007 (1986).

${ }^{14}$ O. L. Alerhand, D. C. Allan, and E. J. Mele, Phys. Rev. Lett. 55, 2700 (1985).

${ }^{15}$ O. L. Alerhand and E. J. Mele, Phys. Rev. Lett. 59, 657 (1987).

${ }^{16}$ F. Ancilotto, A. Selloni, W. Andreoni, S. Baroni, R. Car, and M. Parrinello, Phys. Rev. B 43, 8930 (1991).

${ }^{17}$ P. Chiaradia, A. Cricenti, S. Selci, and G. Chiarotti, Phys. Rev. Lett. 52, 1145 (1984).

${ }^{18}$ C. Goletti, G. Bussetti, F. Arciprete, P. Chiaradia, and G. Chiarotti, Phys. Rev. B 66, 153307 (2002).

${ }^{19}$ R. DelSole and A. Selloni, Phys. Rev. B 30, 883 (1984).

${ }^{20}$ P. Maartensson, A. Cricenti, and G. V. Hansson, Phys. Rev. B 32, 6959 (1985).

${ }^{21}$ D. Straub, L. Ley, and F. J. Himpsel, Phys. Rev. Lett. 54, 142 (1985).
${ }^{22}$ P. Perfetti, J. M. Nicholls, and B. Reihl, Phys. Rev. B 36, 6160 (1987).

${ }^{23}$ G. Bussetti et al., Phys. Rev. Lett. 106, 067601 (2011).

${ }^{24}$ C. H. Patterson, Mol. Phys. 108, 3181 (2010).

${ }^{25}$ R. Dovesi et al., Crystal09 User's Manual (University of Torino, Torino, 2009).

${ }^{26}$ R. I. G. Uhrberg, G. V. Hansson, J. M. Nicholls, and S. A. Flodström, Phys. Rev. Lett. 48, 1032 (1982).

${ }^{27}$ L. Reining and R. DelSole, Phys. Rev. Lett. 67, 3816 (1991).

${ }^{28} \mathrm{M}$. Palummo, N. Witkowski, O. Pluchery, R. DelSole, and Y. Borensztein, Phys. Rev. B 79, 035327 (2009).

${ }^{29}$ C. Goletti, G. Bussetti, P. Chiaradia, and G. Chiarotti, J. Phys. Condens. Matter 16, S4289 (2004).

${ }^{30}$ P. Weightman, D. Martin, R. Cole, and T. Farrell, Rep. Prog. Phys. 68, 1251 (2005).

${ }^{31}$ J. D. E. McIntyre and D. E. Aspnes, Surf. Sci. 24, 417 (1971).

${ }^{32}$ M. K. Kelly, S. Zollner, and M. Cardona, Surf. Sci. 285, 282 (1993).

${ }^{33}$ J. Rath and A. J. Freeman, Phys. Rev. B 11, 2109 (1975).

${ }^{34}$ J. E. Jaffe and A. C. Hess, Phys. Rev. B 48, 7903 (1993).

${ }^{35}$ F. Pascale, M. Catti, A. Damin, R. Orlando, V. Saunders, and R. Dovesi, J. Phys. Chem. B 109, 18522 (2005).

${ }^{36}$ A. D. Becke, J. Chem. Phys. 98, 5648 (1993).

${ }^{37}$ P. J. Stephens, F. J. Devlin, C. F. Chabalowski, and M. J. Frisch, J. Phys. Chem. 98, 11623 (1994).

${ }^{38}$ P. A. M. Dirac, Proc. Cambridge Phil. Soc. 26, 376 (1930).

${ }^{39}$ A. D. Becke, Phys. Rev. A 38, 3098 (1988).

${ }^{40}$ C. Lee, W. Yang, and R. G. Parr, Phys. Rev. B 37, 785 (1988).

${ }^{41}$ S. H. Vosko, L. Wilk, and M. Nusair, Can. J. Phys. 58, 1200 (1980),

${ }^{42}$ F. Pascale, C. Zicovich-Wilson, F. Lopez, B. Civalleri, R. Orlando, and R. Dovesi, J. Comput. Chem. 25, 888 (2004).

${ }^{43}$ C. Zicovich-Wilson, F. Pascale, C. Roetti, V. Saunders, R. Orlando, and R. Dovesi, J. Comput. Chem. 25, 1873 (2004).

${ }^{44}$ S. Dall'Olio, R. Dovesi, and R. Resta, Phys. Rev. B 56, 10105 (1997). 